\title{
Effect of Co-Medication on the Pharmacokinetic Parameters of Phenobarbital in Asphyxiated Newborns
}

\author{
M. ŚÍMA ${ }^{1}$, P. POKORNÁ ${ }^{1,2,3}$, K. HRONOVÁ ${ }^{1}$, O. SLANA $\check{R}^{1}$ \\ ${ }^{1}$ Department of Pharmacology, First Faculty of Medicine, Charles University in Prague and General \\ University Hospital in Prague, Czech Republic, ${ }^{2}$ Department of Pediatrics - PICU/NICU, General \\ University Hospital in Prague and First Faculty of Medicine, Charles University in Prague, Czech \\ Republic, ${ }^{3}$ Intensive Care and Department of Pediatric Surgery, Erasmus MC - Sophia Childrens \\ Hospital, Rotterdam, Netherlands
}

Received September 15, 2015

Accepted October 2, 2015

\section{Summary}

Phenobarbital is an anticonvulsive drug widely used in newborns with hypoxic-ischemic encephalopathy. The objective of our study was to describe possible effect of frequently co-administered medications (dopamine, dobutamine, norepinephrine, furosemide, phenytoin, and analgesics) on the phenobarbital pharmacokinetics in full term newborns with hypoxic-ischemic encephalopathy. Phenobarbital pharmacokinetic parameters (standardized intravenous loading dose was $10-20 \mathrm{mg} / \mathrm{kg}$, maintenance dose 2-6 mg/kg/day) were computed using non-compartmental analysis. Co-medication was evaluated throughout the whole treatment period up to 5 days. Volume of distribution, clearance, and half-life median values (95\% CI) for phenobarbital in the whole study population $(n=37)$ were $0.48(0.41-0.56) \mathrm{l} / \mathrm{kg}, \quad 0.0034 \quad(0.0028-0.0040) \mathrm{l} / \mathrm{h} / \mathrm{kg}$, and 93.7 (88.1-99.2) h, respectively. Phenobarbital pharmacokinetic parameters were not significantly affected by vasoactive drugs (dopamine, dobutamine, and norepinephrine), furosemide, phenytoin, or analgesics. Furthermore, no dose-dependent alteration of phenobarbital pharmacokinetic parameters was noted for vasoactive medication at doses equivalent to cumulative vasoactive-inotropic score (area under the curve in a plot of vasoactive-inotropic score against time) 143.2-8473.6, furosemide at cumulative doses of $0.2-42.9 \mathrm{mg} / \mathrm{kg}$, or phenytoin at cumulative doses of $10.3-46.2 \mathrm{mg} / \mathrm{kg}$. Phenobarbital pharmacokinetics was not affected by investigated co-administered drugs used in newborns with hypoxic-ischemic encephalopathy in real clinical settings.

\section{Key words}

Phenobarbital • Pharmacokinetics • Drug interactions • Newborn - Asphyxia

\section{Corresponding author}

M. Šíma, Department of Pharmacology, First Faculty of Medicine, Charles University in Prague and General University Hospital in Prague, Albertov 4, 12800 Prague 2, Czech Republic. E-mail: sima.mart@seznam.cz

\section{Introduction}

Phenobarbital (PB) is an anticonvulsive drug used as a first line treatment option for control and treatment of seizures in asphyxiated newborns in case of hypoxic-ischemic encephalopathy (HIE) either under therapeutic whole-body hypothermia or without hypothermia (Hall et al. 1998). PB shows highly variable pharmacokinetics, especially distribution and elimination show high inter-individual variability. About $25 \%$ of a PB dose is excreted unchanged, while the major part is metabolized by oxidation via $2 \mathrm{C} 9$ enzyme of cytochrome P450 (CYP). Additionally, CYP2C19, CYP2E1, and $\mathrm{N}$-glucosidation contribute to the drug metabolism to a lesser extent (Kwan and Brodie 2004). PB pharmacokinetics in newborns is different as compared with the adult population; postnatal changes in body composition and maturation have been suggested to alter pharmacokinetics (Alcorn and McNamara 2003). Touw et al. (2000) described the main pharmacokinetic (PK) 
parameters of $\mathrm{PB}$ in neonates as follows: volume of distribution normalized per $\mathrm{kg}$ of body weight $(\mathrm{Vd})$ $0.71(0.21) 1 / \mathrm{kg}$, clearance normalized per $\mathrm{kg}$ of body weight (CL) $0.0043(0.0011) \mathrm{l} / \mathrm{kg} / \mathrm{h}$ and half-life (T1/2) 107.0 (64.0) h. In theory, the drug disposition could also be affected by body temperature (Zanelli et al. 2011), although no clinically relevant effect of moderate therapeutic hypothermia on PB pharmacokinetics has been identified recently (Filippi et al. 2011, van den Broek et al. 2012, Shellhaas et al. 2013).

Since metabolism is the dominant elimination pathway, there is a risk of $\mathrm{PK}$ interactions with concomitantly administered drugs (Patsalos et al. 2008). Although there is a considerably wide armamentarium of drugs that are occasionally used at the pediatric/neonatal intensive care unit (PICU/NICU) departments of which co-administration with $\mathrm{PB}$ could result in a $\mathrm{PK}$ interaction affecting the $\mathrm{PB}$ concentrations, only few drugs/drug classes are often used. Among the frequently used co-medications there is a theoretical risk of PK interaction between $\mathrm{PB}$ and vasoactive drugs (dopamine, dobutamine, and norepinephrine) that could alter the $\mathrm{PB}$ clearance by possible changes in renal perfusion (Schetz 2002). Furosemide could alter the volume of distribution for PB by altered body water content in various compartments (O'Donovan and Bell 1989) and comedication with inducers/inhibitors/competitive inhibitors (phenytoin, sufentanil, midazolam, and tramadol) of cytochrome P450 could alter the metabolic fate of PB in the organism.

This study builds upon our previous work, in which we analyzed the pharmacokinetics of PB in critically ill asphyxiated newborns with the aim to evaluate the role of covariates (age, disease, and therapeutic hypothermia) on the PB pharmacokinetics. The PK parameters in our study were not substantially different from those published previously in similar patient population (Touw et al. 2000), Vd 0.4941 (0.2439) 1/kg, CL 0.0040 (0.0023) 1/h/kg and T1/2 106.55 (59.07) h. However, therapeutic hypothermia did not significantly affect the PB pharmacokinetics in critically ill asphyxiated newborns.

The objective of this study was to describe possible effect of frequently co-administered medications (dopamine, dobutamine, norepinephrine, furosemide, phenytoin, midazolam, sufentanil, and tramadol) on the PB pharmacokinetics in full-term newborns with HIE.

\section{Methods}

\section{Study design}

This was a prospective open-label clinical study that included full term asphyxiated newborns (gestational age $\geq 37$ weeks) with HIE treated with PB. The study was conducted at PICU/NICU of the Department of Pediatrics, General University Hospital and First Faculty of Medicine Charles University in Prague from January 2006 to December 2013. Approval of the study was provided by the Ethics Committee of the General Faculty Hospital, in Prague. Parents of newborns included in the study signed the written informed consent prior to enrollment into the study. Exclusion criteria were neonatal abstinence syndrome, intracranial hemorrhage, severe congenital abnormalities and encephalopathy due to other causes.

Standardized per protocol PB (Luminal inj., Desitin Arzneimitttel GmbH, Hamburg, Germany) dosing consisted from a loading dose of PB 10-20 mg/ $\mathrm{kg} / \mathrm{dose}$ intravenously (iv) administered in $15 \mathrm{~min}$ infusion followed by repeated loading doses of PB to a maximal daily loading dose of $40 \mathrm{mg} / \mathrm{kg} /$ day (Gal et al. 1982). Newborns without clinical or amplitude-integrated electroencephalography (aEEG) response (clinical or subclinical seizures) to the administration of a maximum loading dose of $\mathrm{PB}$ received either phenytoin (Epanutin inj., Hameln Pharmaceuticals GmbH, Hameln, Germany) loading dose of $15-20 \mathrm{mg} / \mathrm{kg}$ iv, followed by maintenance dosing of $2.5-4 \mathrm{mg} / \mathrm{kg}$ iv twice a day or midazolam (Midazolam Torrex inj., Chiesi Pharmaceuticals $\mathrm{GmbH}$, Vienna, Austria) dose of $0.05-0.3 \mathrm{mg} / \mathrm{kg}$ iv as continuous infusion until the end of clinical or aEEG/subclinical seizures. The PB maintenance dose was $1-3 \mathrm{mg} / \mathrm{kg}$ iv administered in $15 \mathrm{~min}$ infusion twice a day (Fischer et al. 1981). Blood samples for PB levels monitoring were to be collected from an arterial line at 2-3, 24, 48, 72, and $96 \mathrm{~h}$ after PB loading dose.

\section{Pharmacokinetic analysis}

Plasma concentrations of phenobarbital (cPB) were measured using a fluorescence polarization immunoassay (FPIA, AxSYM Phenobarbital, Abbott laboratories, Abbott Park, USA) or by quantitative enzyme immunoassay (CEDIA® Phenobarbital II, Microgenics Corporation, Fremont, USA).

Individual PK parameters - Vd and elimination rate constant (Kel) were calculated in a noncompartmental PK model based on individual 
demographic, clinical data and observed cPB using MWPharm 3.01 software (MediWare, Prague, Czech Republic). The PB population PK non-compartment model was individualized to maximize fitting of the simulated PK profile curve with observed concentration points in each patient. Other individual PK parameters $\mathrm{CL}$ and $\mathrm{T} 1 / 2$ - were calculated using following formulae: $\mathrm{CL}=\mathrm{Kel}^{*} \mathrm{Vd}$ and $\mathrm{T} 1 / 2=\ln 2 / \mathrm{Kel}$.

Evaluation of co-medication effect on the $P B$ pharmacokinetics

Any used of co-medication, the time of drug administration, posology and dosing were recorded throughout the study. The primary evaluated comedication were dopamine, dobutamine, norepinephrine, phenytoin, sufentanil, midazolam, tramadol and furosemide, while the other drugs were recorded for exploratory analyses only. To evaluate the cumulative effect of vasoactive compounds (dopamine, dobutamine, and norepinephrine) on the PB pharmacokinetics a standardized vasoactive-inotropic score (VIS) was used VIS $=$ dopamine dose $(\mu \mathrm{g} / \mathrm{kg} / \mathrm{min})+$ dobutamine dose $(\mu \mathrm{g} / \mathrm{kg} / \mathrm{min})+100 *$ norepinephrine dose $(\mu \mathrm{g} / \mathrm{kg} / \mathrm{min})$ (Kumar et al. 2014).

Both possible dose-dependent and doseindependent interactions between $\mathrm{PB}$ and the comedication were evaluated. To detect the dose-dependent interactions, cumulative doses of co-medication within acute phase of treatment normalized per $\mathrm{kg}$ of body weight were used, while for dose-independent interactions any dosing of co-administered compound was considered.

\section{Statistical analysis}

Descriptive parameters means, standard deviations (SD) and medians of PB PK parameters were calculated using GraphPad Prism 3.02 (GraphPad Software, Inc., La Jolla, USA). The $95 \%$ confidence intervals $(\mathrm{CI})$ for medians were calculated by method of Bonett and Price (2002).

Linear regression models and Mann-Whitney $\mathrm{U}$ test were used to evaluate the impact of co-medication on PB PK parameters using GraphPad Prism 3.02 (GraphPad Software, Inc., La Jolla, USA).

Table 1. Proportion of patients using specific co-medication.

\begin{tabular}{cccccc}
\hline & $\begin{array}{c}\text { Any } \\
\text { co-medication }\end{array}$ & $\begin{array}{c}\text { Any } \\
\text { vasopressor }\end{array}$ & Dopamine & Dobutamine & Norepinephrine \\
\hline$n / N$ & $37 / 37$ & $32 / 37$ & $31 / 37$ & $30 / 37$ & $4 / 37$ \\
$(\%)$ & $(100.00)$ & $(86.49)$ & $(83.78)$ & $(81.08)$ & $(10.81)$ \\
\hline & Phenytoin & Sufentanil & Midazolam & Tramadol & Furosemide \\
\hline$n / N$ & $11 / 37$ & $27 / 37$ & $26 / 37$ & $14 / 37$ & $26 / 37$ \\
$(\%)$ & $(29.73)$ & $(72.97)$ & $(70.27)$ & $(37.84)$ & $(70.27)$ \\
\hline
\end{tabular}

\section{Results}

Thirty seven full term newborns were enrolled to the study (22 males, 15 females); 24 patients were treated under full body hypothermia, while normothermic conditions were applied in 13 patients. Mean (SD) gestational age in the study population was 39.32 (1.36) weeks, body weight $3.24(0.65) \mathrm{kg}$. PB loading dose ranged from 5.04 to $34.29 \mathrm{mg} / \mathrm{kg}$ body weight and the maintenance dose ranged between 1.07 and 20.31 $\mathrm{mg} / \mathrm{kg} /$ day.

Totally $110 \mathrm{cPB}$ points for pharmacokinetics analysis were obtained (2-5 cPB points per patient). Mean (SD) $\mathrm{Vd}, \mathrm{CL}$, and $\mathrm{T} 1 / 2$ values for $\mathrm{PB}$ were 0.4941
$(0.2439) \mathrm{l} / \mathrm{kg}, \quad 0.0040 \quad(0.0023) \mathrm{l} / \mathrm{h} / \mathrm{kg}, \quad$ and $\quad 106.55$ (59.07) h, respectively. There was high inter-individual variability of all PK parameters in our study population indicated by coefficient of variation of $49.35 \%, 58.00 \%$, and $55.44 \%$ for $\mathrm{Vd}, \mathrm{CL}$, and $\mathrm{T} 1 / 2$, respectively.

Distribution of the use of co-administered compounds in the study population is summarized in the Table 1 and comparison of PB PK parameters in subgroups with and without co-medication is shown in the Table 2 .

There were no significant dose-dependent drugdrug interactions affecting PB pharmacokinetics between the studied drugs and $\mathrm{PB}$ as indicated by linear regression between PB PK parameters and cumulative doses of 
co-medication normalized per $\mathrm{kg}$ of body weight. Similarly, linear regression between PB PK parameters and VIS showed no significant relationship (Fig. 1). We did not observe significant dose-independent interactions between co-administered drugs and PB (Table 2). There was significantly decreased $\mathrm{CL}$ among patients treated with dopamine as compared with the patient subgroup without dopamine treatment $(\mathrm{P}=0.0246)$.

Table 2. Medians (95\% CI) of phenobarbital pharmacokinetic parameters in subgroups with $(\mathrm{Y})$ and without (N) co-medication.

\begin{tabular}{|c|c|c|c|c|}
\hline & & Vd (l/kg) & CL (l/h/kg) & T1/2 (h) \\
\hline All patients & & $\begin{array}{c}0.4837 \\
(0.4065-0.5609)\end{array}$ & $\begin{array}{c}0.0034 \\
(0.0028-0.0040)\end{array}$ & $\begin{array}{c}93.65 \\
(88.08-99.21)\end{array}$ \\
\hline Any vasoactive (inotropic) drug & $\mathrm{N}$ & $\begin{array}{c}0.4703 \\
(0.3670-0.5736) \\
0.4884 \\
(0.3423-0.6345)\end{array}$ & $\begin{array}{c}0.0034 \\
(0.0028-0.0040) \\
0.0043 \\
(0.0032-0.0055)\end{array}$ & $\begin{array}{c}93.02 \\
(73.88-112.17) \\
93.65 \\
(66.58-120.72)\end{array}$ \\
\hline Dopamine & $\mathrm{N}$ & $\begin{array}{c}0.4468 \\
(0.3447-0.5489) \\
0.5389 \\
(0.4138-0.6639)\end{array}$ & $\begin{array}{c}0.0033^{*} \\
(0.0026-0.0040) \\
0.0044 \\
(0.0035-0.0052)\end{array}$ & $\begin{array}{c}93.65 \\
(70.83-116.47) \\
93.02 \\
(78.96-107.09)\end{array}$ \\
\hline Dobutamine & Y & $\begin{array}{c}0.4703 \\
(0.2660-0.5746) \\
0.4884 \\
(0.3759-0.6009)\end{array}$ & $\begin{array}{c}0.0034 \\
(0.0026-0.0042) \\
0.0043 \\
(0.0035-0.0052)\end{array}$ & $\begin{array}{c}93.02 \\
(73.38-112.67) \\
93.65 \\
(72.79-114.50)\end{array}$ \\
\hline Norepinephrine & $\mathrm{N}$ & $\begin{array}{c}0.4428 \\
(0.1355-0.7501) \\
0.4837 \\
(0.4109-0.5565)\end{array}$ & $\begin{array}{c}0.0052 \\
(0.0028-0.0075) \\
0.0034 \\
(0.0029-0.0039)\end{array}$ & $\begin{array}{c}62.78 \\
(-94.89-220.45) \\
93.65 \\
(79.82-107.48)\end{array}$ \\
\hline Phenytoin & $\mathrm{N}$ & $\begin{array}{c}0.4569 \\
(0.2779-0.6359) \\
0.4841 \\
(0.3614-0.6067)\end{array}$ & $\begin{array}{c}0.0034 \\
(0.0020-0.0047) \\
0.0035 \\
(0.0026-0.0044)\end{array}$ & $\begin{array}{c}128.33 \\
(97.04-159.63) \\
92.40 \\
(82.15-102.65)\end{array}$ \\
\hline Sufentanil & $\mathrm{N}$ & $\begin{array}{c}0.4844 \\
(0.3802-0.5886) \\
0.4703 \\
(0.2984-0.6422)\end{array}$ & $\begin{array}{c}0.0034 \\
(0.0024-0.0043) \\
0.0039 \\
(0.0031-0.0048)\end{array}$ & $\begin{array}{c}93.65 \\
(74.32-112.97) \\
93.02 \\
(65.18-120.87)\end{array}$ \\
\hline Midazolam & $\mathrm{N}$ & $\begin{array}{c}0.4841 \\
(0.3716-0.5965) \\
0.4569 \\
(0.2663-0.6475)\end{array}$ & $\begin{array}{c}0.0034 \\
(0.0025-0.0043) \\
0.0037 \\
(0.0026-0.0048)\end{array}$ & $\begin{array}{c}93.02 \\
(87.96-98.09) \\
99.00 \\
(64.55-133.45)\end{array}$ \\
\hline Tramadol & $\mathrm{N}$ & $\begin{array}{c}0.4841 \\
(0.3314-0.6367) \\
0.4569 \\
(0.3567-0.5571)\end{array}$ & $\begin{array}{c}0.0034 \\
(0.0024-0.0043) \\
0.0037 \\
(0.0026-0.0048)\end{array}$ & $\begin{array}{c}99.00 \\
(50.13-147.87) \\
92.40 \\
(85.88-98.92)\end{array}$ \\
\hline Furosemide & Y & $\begin{array}{c}0.4844 \\
(0.3547-0.6141) \\
0.4519 \\
(0.3137-0.5900)\end{array}$ & $\begin{array}{c}0.0034 \\
(0.0024-0.0044) \\
0.0035 \\
(0.0024-0.0046)\end{array}$ & $\begin{array}{c}92.40 \\
(60.67-124.13) \\
93.65 \\
(86.86-100.44)\end{array}$ \\
\hline
\end{tabular}

$* p<0.05$ vs. group without co-administered drug. 


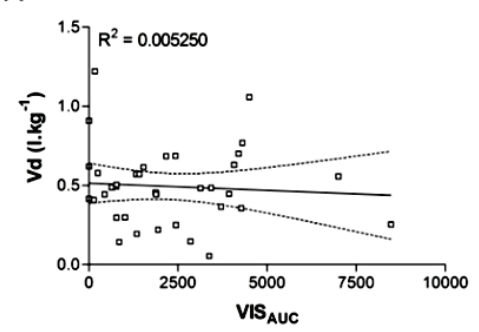

D

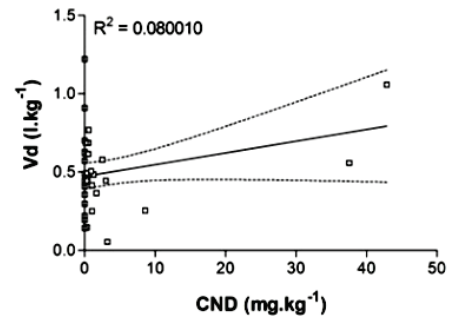

G

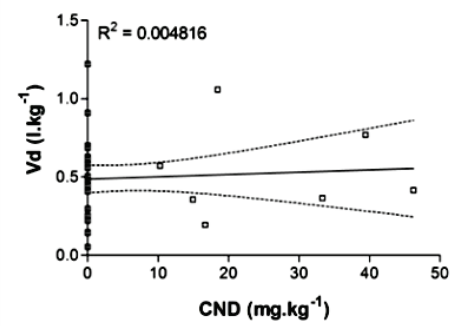

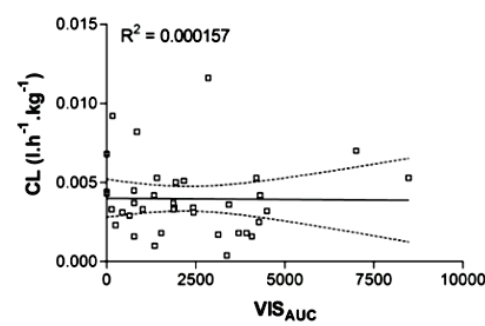

$E$

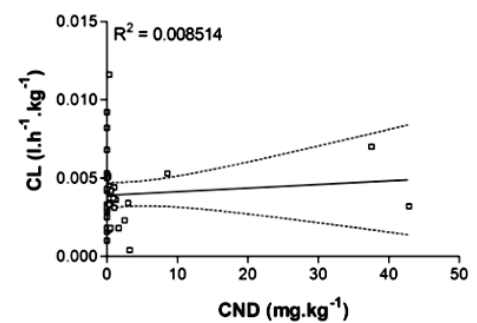

$\mathrm{H}$

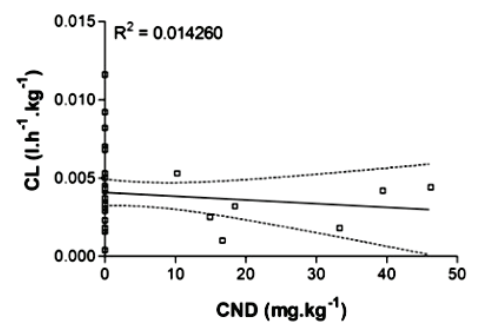

C

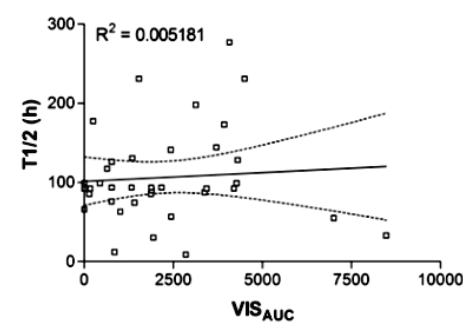

$\mathrm{F}$
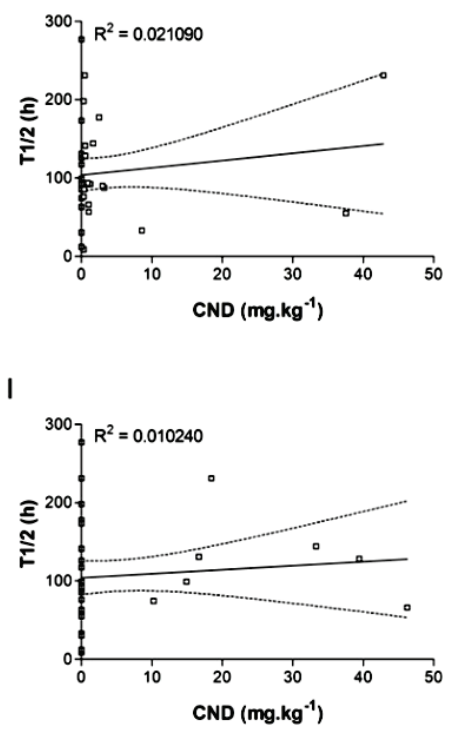

Fig. 1. A. Relationship between phenobarbital volume of distribution and vasoactive-inotropic score; B. Relationship between phenobarbital clearance and vasoactive-inotropic score; C. Relationship between phenobarbital half-life and vasoactive-inotropic score; D. Relationship between phenobarbital volume of distribution and furosemide cumulative dose; $\mathbf{E}$. Relationship between phenobarbital clearance and furosemide cumulative dose; F. Relationship between phenobarbital half-life and furosemide cumulative dose; G. Relationship between phenobarbital volume of distribution and phenytoin cumulative dose; $\mathbf{H}$. Relationship between phenobarbital clearance and phenytoin cumulative dose; I. Relationship between phenobarbital half-life and phenytoin cumulative dose. CND is cumulative dose of co-medication normalized per $\mathrm{kg}$ of body weight. VIS $_{\text {Auc }}$ is the area under the curve in a plot of vasoactive-inotropic score against time. $R^{2}$ is coefficient of determination.

\section{Discussion}

This study was conducted to evaluate, if there are any clinically relevant drug-drug interactions affecting PB pharmacokinetics caused due to frequently co-administered compounds in critically ill newborns with HIE.

We focused on three theoretically possible mechanistic pathways for these PK interactions, i.e. alteration of renal blood flow after vasoactive medications, changes in body water content in numerous compartments induced by diuretics, and also alterations in elimination due to alterations of liver drug metabolism.

Since norepinephrine may induce decreased renal blood flow, while low-dose dopamine counteract this effect (Richer et al. 1996), there is a theoretical concern that the vasoactive medication may alter PB clearance due to the changes in renal blood flow.
Therefore we examined the effect of vasoactive drugs (dopamine, dobutamine, and norepinephrine) that are frequently used in this patient population. However, there was no apparent interaction between vasoactive medication and $\mathrm{PB}$, although statistical analysis has indicated dose-independent difference in $\mathrm{CL}$ of $\mathrm{PB}$ between the subgroups with and without dopamine. When we critically analyzed this finding and we also took into account the limitations of our study, we came to a conclusion that this is most likely an artifact. Firstly, there was no clear trend in dose-dependency of the possible dopamine-PB interaction. Secondly, neither the other vasoactive compounds nor VIS affected PB clearance. Further, the patient population is very specific and difficult for conducting any clinical study in it, but the sample size is rather limited as also indicated that there were only 6 patients in the non-dopamine group.

While it was previously shown that furosemide 
significantly decreases total body water, extracellular water, and interstitial water (O'Donovan and Bell 1989) no possibly expected effect on $\mathrm{PB}$ volume of distribution was seen in this study.

We also explored the possible effect of comedication with possible effects on $\mathrm{PB}$ metabolic elimination via cytochrome P450 (phenytoin, sufentanil, midazolam, and tramadol). Phenytoin could interact with $\mathrm{PB}$ either as an inducer or an inhibitor of metabolism, depending on the length of treatment with the combination of both drugs as suggested previously (Encinas et al. 1992). An increase in PB plasma levels during the initial phase of concomitant treatment with phenytoin due to competition on same metabolic pathways (CYP2C9, CYP2C19) could be expected (Patsalos et al. 2008). On the contrary, longer treatment could result in subsequently decreased PB plasma levels after phenytoin-induced synthesis of CYP enzymes via activation of nuclear receptors (pregnane $\mathrm{X}$ receptor, constitutive androstane receptor) (Brodie et al. 2013). However, these interactions have not been observed in our study, similarly as with the other co-administered compounds, although the drug interactions with sufentanil, midazolam, and tramadol were less likely, because these drugs are dominantly metabolized by other enzymes than PB (CYP3A4, CYP2D6) and do not represent strong enzyme inducers/inhibitors (Gorski et al. 1994, Tateishi et al. 1996, Subrahmanyam et al. 2001). Overall, the lack of observed metabolic interactions may be due to the short time of concomitant treatment in real clinical settings and/or due to the fact that there are multiple elimination pathways involved in $\mathrm{PB}$ metabolism and elimination (namely renal excretion of parent substance, metabolism via CYP2C9, CYP2C19, CYP2E1 and N-glucosidation), where alteration of one elimination pathway may be compensated by another one.

During an exploratory analysis, no interaction between PB and other rather erratically co-administered drugs was seen, similarly as there was no apparent effect of therapeutic whole body hypothermia, although the study population size is too small for proper analysis of these possible confounding factors. Most patients $(94.59 \%)$ were treated by two or more co-administered drugs simultaneously. Except the VIS no synergistic effect of two or more co-administered drugs was analyzed due to low patient number in each subgroup.

Observed high variability of PB PK parameters suggests a suitability of routine therapeutic drug monitoring of PB in newborns with HIE.

We acknowledge that there is a slight limitation of our study resulting from the fact that it was carried out in naturalistic clinical settings and thus there were number of protocol deviations mainly with respect to $\mathrm{PB}$ dosing and the blood sample collection times for cPB analyses. However, we did not exclude the patients with these deviations from PK analyses, since the protocol differences were compensated by individualized analysis of PB PK parameters that took the deviations from per protocol doses or sample times into account.

In conclusion, this study did not show clinically relevant effects of frequently used co-medication on $\mathrm{PB}$ pharmacokinetics in newborns with HIE.

\section{Conflict of Interest}

There is no conflict of interest.

\section{Acknowledgements}

The work was supported by the Charles University Project PRVOUK P25/LF1/2.

\section{References}

ALCORN J, MCNAMARA PJ: Pharmacokinetics in the newborn. Adv Drug Deliv Rev 55: 667-686, 2003.

BONETT DG, PRICE RM: Statistical inference for a linear function of medians: confidence intervals, hypothesis testing, and sample size requirements. Psychol Methods 7: 370-383, 2002.

BRODIE MJ, MINTZER S, PACK AM, GIDAL BE, VECHT CJ, SCHMIDT D: Enzyme induction with antiepileptic drugs: cause for concern? Epilepsia 54: 11-27, 2013.

ENCINAS MP, SANTOS BUELGA D, ALONSO GONZÁLEZ AC, GARCÍA SÁNCHEZ MJ, DOMÍNGUEZ-GIL HURLÉ A: Influence of length of treatment on the interaction between phenobarbital and phenytoin. $J$ Clin Pharm Ther 17: 49-50, 1992.

FILIPPI L, LA MARCA G, CAVALLARO G, FIORINI P, FAVELLI F, MALVAGIA S, DONZELLI G, GUERRINI R: Phenobarbital for neonatal seizures in hypoxic ischemic encephalopathy: a pharmacokinetic study during whole body hypothermia. Epilepsia 52: 794-801, 2011. 
FISCHER JH, LOCKMAN LA, ZASKE D, KRIEL R: Phenobarbital maintenance dose requirements in treating neonatal seizures. Neurology 31: 1042-1044, 1981.

GAL P, BOER HR, TOBACK J, ERKAN NV: Phenobarbital dosing in neonates and asphyxia. Neurology 32: 788-789, 1982.

GORSKI JC, HALL SD, JONES DR, VANDENBRANDEN M, WRIGHTON SA: Regioselective biotransformation of midazolam by members of the human cytochrome P450 3A (CYP3A) subfamily. Biochem Pharmacol 47: 1643-1653, 1994.

HALL RT, HALL FK, DAILY DK: High-dose phenobarbital therapy in term newborn infants with severe perinatal asphyxia: a randomized, prospective study with three-year follow-up. J Pediatr 132: 345-348, 1998.

KUMAR M, SHARMA R, SETHI SK, BAZAZ S, SHARMA P, BHAN A, KHER V: Vasoactive Inotrope Score as a tool for clinical care in children post cardiac surgery. Indian J Crit Care Med 18: 653-658, 2014.

KWAN P, BRODIE MJ: Phenobarbital for the treatment of epilepsy in the 21 st century: a critical review. Epilepsia 45 : 1141-1149, 2004.

O'DONOVAN BH, BELL EF: Effects of furosemide on body water compartments in infants with bronchopulmonary dysplasia. Pediatr Res 26: 121-124, 1989.

PATSALOS PN, BERRY DJ, BOURGEOIS BF, CLOYD JC, GLAUSER TA, JOHANNESSEN SI, LEPPIK IE, TOMSON T, PERUCCA E: Antiepileptic drugs--best practice guidelines for therapeutic drug monitoring: a position paper by the subcommission on therapeutic drug monitoring, ILAE Commission on Therapeutic Strategies. Epilepsia 49: 1239-1276, 2008.

RICHER M, ROBERT S, LEBEL M: Renal hemodynamics during norepinephrine and low-dose dopamine infusions in man. Crit Care Med 24: 1150-1156, 1996.

SCHETZ M: Vasopressors and the kidney. Blood Purif 20: 243-251, 2002.

SHELLHAAS RA, NG CM, DILLON CH, BARKS JD, BHATT-MEHTA V: Population pharmacokinetics of phenobarbital in infants with neonatal encephalopathy treated with therapeutic hypothermia. Pediatr Crit Care Med 14: 194-202, 2013.

SUBRAHMANYAM V, RENWICK AB, WALTERS DG, YOUNG PJ, PRICE RJ, TONELLI AP, LAKE BG: Identification of cytochrome P-450 isoforms responsible for cis-tramadol metabolism in human liver microsomes. Drug Metab Dispos 29: 1146-1155, 2001.

TATEISHI T, KRIVORUK Y, UENG YF, WOOD AJ, GUENGERICH FP, WOOD M: Identification of human liver cytochrome P-450 3A4 as the enzyme responsible for fentanyl and sufentanil N-dealkylation. Anesth Analg 82: 167-172, 1996.

TOUW DJ, GRAAFLAND O, CRANENDONK A, VERMEULEN RJ, VAN WEISSENBRUCH MM: Clinical pharmacokinetics of phenobarbital in neonates. Eur J Pharm Sci 12: 111-116, 2000.

VAN DEN BROEK MP, GROENENDAAL F, TOET MC, VAN STRAATEN HL, VAN HASSELT JG, HUITEMA AD, DE VRIES LS, EGBERTS AC, RADEMAKER CM: Pharmacokinetics and clinical efficacy of phenobarbital in asphyxiated newborns treated with hypothermia: a thermopharmacological approach. Clin Pharmacokinet 51: 671-679, 2012.

ZANELLI S, BUCK M, FAIRCHILD K: Physiologic and pharmacologic considerations for hypothermia therapy in neonates. J Perinatol 31: 377-386, 2011. 